\title{
COUPLING BETWEEN THERMAL OSCILLATIONS IN THE SURFACE OF A MICRO-CYLINDER AND VORTEX SHEDDING
}

\author{
B. Linares, A. Velazquez, and J.L. Montanes \\ Propulsion and Fluid Mechanics Department, School of Aeronautics, Universidad \\ Politecnica de Madrid, Spain
}

\begin{abstract}
This article studies the coupling between prescribed thermal oscillations in the surface of a micro-cylinder and vortex shedding. We deal with the unsteady, laminar, compressible flow regime where the aerodynamics forces have a periodic behavior. It is shown that appropriate spatial and time-dependent temperature oscillations on the surface of the micro-cylinder create a resonance that controls the amplitude and frequency of both lift and drag coefficients. In practice, what we study is a mechanism to modulate the amplitude and frequency of mechanical loads of aerodynamics origin in a micro-structure by using surface temperature fluctuations as the control parameter.
\end{abstract}

KEY WORDS: resonance, thermal oscillation, vortex shedding

\section{INTRODUCTION}

One reason why MEMS are attractive from the engineering point of view is that they allow for a highly integrated level of actuation. For instance, a mesoscale actuator such as, for instance, a positioning system, converts electric impulses into mechanical force and displacement by using a dedicated transformation device; i.e., the electric motor. In some cases, additional subsystems like hydraulic networks are also required to achieve the actuator practical objectives. On the contrary, a distinctive feature of the microscale is that conversion between the electric, thermal, fluid, and mechanical modes is achieved in a much more direct way. A wide spectrum of examples of this type has been recently presented by Pelesko and Bernstein [1], where the authors address coupled thermo-elastic systems, electrostatic-elastic systems, magnetically actuated micro-fluidic devices such as micro-pumps, et cetera. A comprehensive review of the different aspects associated to the analysis and simulation of these integrated systems has been provided by Kirby et al. [2]. 


\begin{tabular}{|c|c|c|c|}
\hline \multicolumn{4}{|c|}{ NOMENCLATURE } \\
\hline$a$ & coefficient in the temperature & $x$ & dimensionless horizontal \\
\hline$b$ & $\begin{array}{l}\text { boundary condition definition } \\
\text { coefficient in the temperature }\end{array}$ & $y$ & $\begin{array}{l}\text { coordinate } \\
\text { dimensionless vertical }\end{array}$ \\
\hline & boundary condition definition & & coordinate \\
\hline$C d$ & drag coefficient: $2 f / \rho_{\infty} u_{\infty}^{2} D$ & & \\
\hline$C d_{\mathrm{av}}$ & average drag coefficient & \multicolumn{2}{|c|}{ Greek Symbols } \\
\hline $\mathrm{Cl}^{-}$ & lift coefficient: $2 f_{y} / \rho_{\infty} u_{\infty}^{2} D$ & $\Delta$ & increment \\
\hline Cl_max & maximum of the lift coefficient & $\gamma$ & specific heat ratio \\
\hline & $\begin{array}{l}\text { coefficient in the temperature } \\
\text { boundary condition definition }\end{array}$ & $\delta_{2 \mathrm{p}}$ & $\begin{array}{l}\text { second-order artificial } \\
\text { dissipation parameter in the }\end{array}$ \\
\hline$D$ & cylinder diameter & & continuity equation \\
\hline$d$ & $\begin{array}{l}\text { typical distance inside a cloud of } \\
\text { points }\end{array}$ & $\delta_{4 \mathrm{p}}$ & $\begin{array}{l}\text { fourth-order artificial } \\
\text { dissipation parameter in the }\end{array}$ \\
\hline$F$ & coefficient in the temperature & & continuity equation \\
\hline & boundary condition definition & $\delta_{4 \mathrm{~T}}$ & fourth-order artificial \\
\hline$f_{x}$ & $\begin{array}{l}\text { horizontal force on the micro- } \\
\text { cylinder }\end{array}$ & & $\begin{array}{l}\text { dissipation parameter in the } \\
\text { energy equations }\end{array}$ \\
\hline$f_{j^{\prime}}^{\prime}$ & $\begin{array}{l}\text { vertical force on the micro- } \\
\text { cylinder }\end{array}$ & $\delta_{4 \mathrm{u}}$ & $\begin{array}{l}\text { fourth-order artificial } \\
\text { dissipation parameter in the }\end{array}$ \\
\hline$L$ & artificial dissipation operator & & momentum equations \\
\hline$M$ & upstream Mach number: & $\theta$ & azimuth angle \\
\hline & $u_{\infty} /\left(\gamma R_{\text {gas }} T_{\infty}\right)^{1 / 2}$ & $\lambda_{0}-\lambda_{5}$ & coefficients in the least squares \\
\hline $\mathrm{Pr}$ & Prandtl number & & approximation \\
\hline $\operatorname{Re}$ & upstream Reynolds number: & $\mu$ & fluid viscosity \\
\hline & $\rho_{\infty} u_{-\infty} D / \mu_{\infty}$ & $\xi$ & combination of artificial \\
\hline$\stackrel{S}{T}$ & $\begin{array}{l}\text { Strouhal number: } 1 / \tau_{d_{-}} \text {cvcle } \\
\text { dimensionless temperature }\end{array}$ & & $\begin{array}{l}\text { dissipation and discretization } \\
\text { parameters }\end{array}$ \\
\hline$T_{\propto}$ & upstream temperature & $\rho$ & dimensionless density \\
\hline$t$ & dimensionless time & $\rho_{x}$ & upstream density \\
\hline$u$ & $\begin{array}{l}\text { dimensionless horizontal } \\
\text { velocity }\end{array}$ & $\tau_{d}-v c l e$ & $\begin{array}{l}\text { dimensionless time needed for } \\
\text { the } C l \text { to complete one cycle }\end{array}$ \\
\hline $\begin{array}{l}u_{x} \\
p\end{array}$ & $\begin{array}{l}\text { upstream horizontal velocity } \\
\text { dimensionless vertical velocity }\end{array}$ & $\Phi$ & $\begin{array}{l}\text { representation for either } \rho, u, v \text {. } \\
\text { or } T\end{array}$ \\
\hline
\end{tabular}

The analysis of the coupling between fluid flow and heat transfer from a cylinder has been addressed extensively in the literature. Iwai et al. [3] have studied experimentally and numerically the heat transfer from a circular cylinder when the incoming flow field has a low-frequency oscillating character. They concluded that both heat transfer enhancement and reduction occur during a given cycle and that they counterbalance each other on the average. The problem of convection from a rotating cylinder located in a uniform stream has been analyzed by Mahfouz and Badr [4]. These authors found that there is a frequency lock-on phenomenon that enhances heat transfer significantly. The effect that streamwise oscillations have on heat transfer from a heated cylinder has been addressed by Gau et al. [5], who pointed out that synchronization occurs and that it enhances heat transfer by a sizable amount. The relation between the Reynolds and Strouhal numbers when dealing with the flow that moves around a heated cylinder at constant wall temperature has been analyzed experimentally by Wanget al. [6]. In particular, they propose a novel effective temperature concept that allows for generalization of their results. 
Other examples of coupling between flow oscillation and heat transfer have been provided by Zhang et al. [7] and Ramos et al. [8]. The former deals with a heated oscillating plate, while the later addresses boundary layer flow in the presence of a flat wall having surface temperature space variations. Finally, an interesting fluid coupling study that also involves a magnetizing force has been recently published by Kang and Hyun [9]. References cited previously deal with the incompressible flow regime and, except for the study presented by Ramos et al. [8], wall temperature is assumed to be constant. Guo and $\mathrm{Wu}[10,11]$ have analyzed heat transfer in a microtube when compressibility effects are important. More recently, Linares and Velazquez [12] have studied how different values of constant heat transfer in the surface of a micro-cylinder affect lift and drag in the unsteady, laminar, compressible limit. In particular, they found that lift is rather sensitive to cylinder wall heat flux and that it could be nearly halved for some specific heating rates.

The present work goes a step beyond the scope of Linares and Velazquez [12]. We now study whether a prescribed spatial and temporal thermal oscillation in the surface of a micro-cylinder could create a resonance with the vortex shedding phenomenon to control the extent of aerodynamics forces. Also, we analyze whether the vortex shedding frequency could be modified by using the same control parameter and whether it is feasible to change it from one cycle to the next while keeping constant the frequency of the temperature pulsation. Regarding practical implementation of the coupling effect, Dobrovolsky et al. [13] have shown that it is possible to generate high-amplitude, high-frequency temperature oscillations (up to $3 \mathrm{MHz}$ ) in silicon-on-insulator structures. In our study, we consider vortex shedding frequencies in the range from 10 to $100 \mathrm{KHz}$, which is below the threshold described in Dobrovolsky et al. [13].

The following section describes the problem under consideration and the governing equations and boundary conditions. A brief description of the space and time integration schemes is presented next, and it is followed by a discussion on the artificial dissipation terms used in the simulation and their boundary conditions. The next section deals with solver validation and sensitivity of the results with regard to several computational parameters. Then, the results obtained under a variety of conditions are presented and conclusions are given.

\section{DESCRIPTION OF THE PROBLEM, GOVERNING EQUATIONS AND BOUNDARY CONDITIONS}

We restrict ourselves to the compressible, laminar, unsteady free stream regime and consider ideal fluid properties. In particular, we look for the prescribed spatial and temporal surface temperature oscillations that resonate with the natural frequency of vortex shedding. In this way, we try to enhance or diminish the amplitude of the aerodynamics forces and intend to exert some control on the vortex shedding frequency. The flow parameters that we consider in our study are Mach 0.4 and Reynolds 150 . It will be shown in the results section that the associated vortex shedding Strouhal number is in the range from 0.17 to 0.18 . In these conditions, if we consider the flow of air in the following range-temperature from 300 to $600 \mathrm{~K}$ and density from 0.1 to $0.01 \mathrm{~kg} / \mathrm{m}^{3}$ - we end up with cylinder diameters in the range from $200 \mu \mathrm{m}$ to $2 \mathrm{~mm}$ and vortex shedding frequencies of the order of 10 to $100 \mathrm{KHz}$. For all 
these cases, the Knudsen number (ratio of the gas mean free path to the characteristic length of the problem taken as the micro-cylinder diameter) is smaller than 0.01 , thereby justifying the assumption of continuous medium.

Even though the equations and boundary conditions of the problem have been described in Linares and Velazquez [12], they are repeated here for the sake of completion.

Continuity:

$$
\frac{\partial \rho}{\partial t}+\rho \frac{\partial u}{\partial x}+u \frac{\partial \rho}{\partial x}+\rho \frac{\partial \nu}{\partial y}+\nu \frac{\partial \rho}{\partial y}=0
$$

Momentum:

$$
\begin{gathered}
\frac{\partial u}{\partial t}+u \frac{\partial u}{\partial x}+\nu \frac{\partial u}{\partial y}=-\frac{1}{\gamma M^{2}}\left(\frac{\partial T}{\partial x}+\frac{T}{\rho} \frac{\partial \rho}{\partial x}\right)+\frac{1}{\rho \operatorname{Re}}\left(2 \frac{\partial^{2} u}{\partial x^{2}}+\frac{\partial^{2} u}{\partial y^{2}}+\frac{\partial^{2} \nu}{\partial x \partial y}\right) \\
\frac{\partial \nu}{\partial t}+u \frac{\partial \nu}{\partial x}+\nu \frac{\partial \nu}{\partial y}=-\frac{1}{\gamma M^{2}}\left(\frac{\partial T}{\partial y}+\frac{T}{\rho} \frac{\partial \rho}{\partial y}\right)+\frac{1}{\rho \operatorname{Re}}\left(2 \frac{\partial^{2} \nu}{\partial y^{2}}+\frac{\partial^{2} \nu}{\partial x^{2}}+\frac{\partial^{2} \nu}{\partial x \partial y}\right)
\end{gathered}
$$

Internal energy:

$$
\begin{aligned}
& \frac{\partial T}{\partial t}+u \frac{\partial T}{\partial x}+\nu \frac{\partial T}{\partial y}=(1-\gamma) T\left(\frac{\partial u}{\partial x}+\frac{\partial \nu}{\partial y}\right)+\frac{\gamma}{\rho \operatorname{Re} \operatorname{Pr}}\left(\frac{\partial^{2} T}{\partial x^{2}}+\frac{\partial^{2} T}{\partial y^{2}}\right)+ \\
& +\gamma(\gamma-1) \frac{M^{2}}{\rho \operatorname{Re}}\left[2\left(\frac{\partial u}{\partial x}\right)^{2}+2\left(\frac{\partial \nu}{\partial y}\right)^{2}+\left(\frac{\partial u}{\partial y}+\frac{\partial \nu}{\partial x}\right)^{2}\right]
\end{aligned}
$$

Dimensionless variables $\rho, t, v, T, x, y$, and $t$ are density, horizontal and vertical velocity, temperature, spatial coordinates, and time, respectively. Variables are rendered dimensionless by using the unperturbed upstream density $\rho_{\alpha}$, horizontal velocity $u_{\infty}$, temperature $T_{\infty_{\infty}}$, and cylinder diameter $D . \gamma$ is the specific heat ratio, $M$ the upstream Mach number, Re the Reynolds number based on $D$, and Pr the Prandtl number. State equation has been used to remove pressure from the momentum and energy equations. Boundary conditions are

$$
\begin{gathered}
\text { Inflow section : } u=1, \nu=0, \frac{\partial^{2} \rho}{\partial n^{2}}=0, T=1 \\
\text { outflow section }: \frac{\partial u}{\partial n}=0, \frac{\partial \nu}{\partial n}=0, \frac{\partial \rho}{\partial n}=0, \frac{\partial T}{\partial n}=0 \\
\text { Cylinder wall : } u=0, \nu=0, T=T_{w a l l}(\theta, t)
\end{gathered}
$$

where $n$ is the normal to the considered section and $\theta$ is the azimuthal angle along the micro-cylinder wall measured counterclock wise (see Figure 1). Density $\rho$ at the wall is computed by solving the continuity equation by using one-side derivatives [14]. 

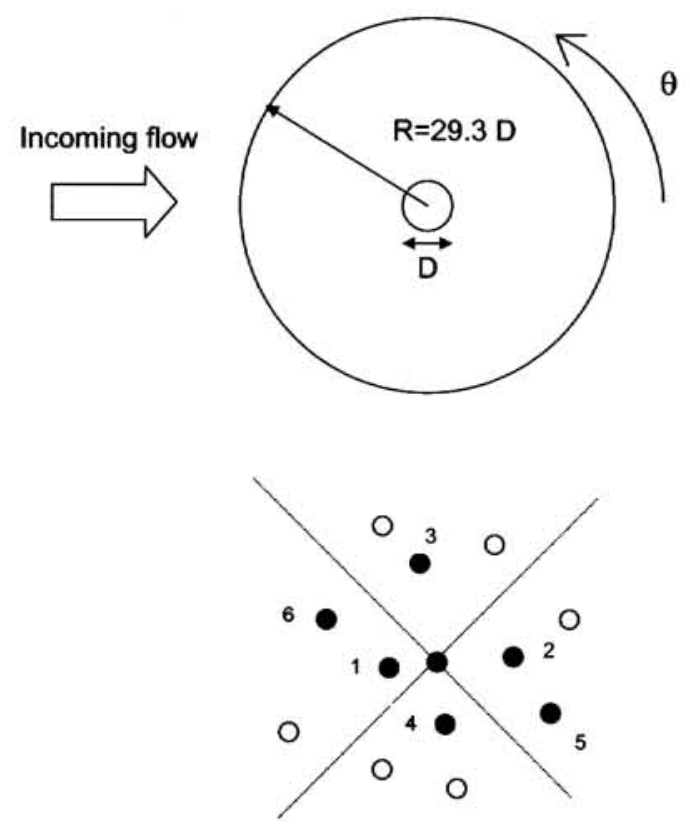

Figure 1. Computational domain and basic cloud configuration.

\section{SPACE AND TIME INTEGRATION}

We follow a finite point approach, similar to the one presented in Mendez and Velazquez $[15,16]$ for incompressible flows, where local functional approximations of the variables are second-order Taylor polynomials:

$$
\Phi(x, y)=\lambda_{0}+\lambda_{1} x+\lambda_{2} y+\lambda_{3} x^{2}+\lambda_{4} y^{2}+\lambda_{5} x y, \Phi \equiv \rho, u, \nu, T
$$

The coefficients $\lambda_{i}$ are computed for each variable by using a least squares approximation in a cloud of six points located around the central one. Four out of these six points are chosen such that they are closest to the centrum and spanning the surrounding space as evenly as feasible. The fifth and sixth points are selected at random (see Figure 1). This random selection has some beneficial global effects: (a) it avoids global skewdness in the computation of the derivatives, (b) it has some stabilizing effects on the convergence process, and (c) it automatically generates the perturbation needed to start the vortex shedding process without the need for other numerical means. Time integration is explicit and follows a standard Lax-Wendroff scheme:

$$
\Phi_{i}^{n+1}=\Phi_{i}^{n}+\left(\frac{\partial \Phi}{\partial t}\right)_{t}^{n} \Delta t+\frac{1}{2}\left(\frac{\partial^{2} \Phi}{\partial t^{2}}\right)_{t}^{n} \Delta t^{2}, \Phi \equiv \rho, u, \nu, T
$$

where $\Delta t$ is the numerical time integration step. The second-order derivative in expression (9) is evaluated by performing an additional time derivative in eqs. 1-4 leaving out the terms that contain second-order spatial derivatives. Finite point formulations are well 
suited to be used in combination with Lax-Wendroff time integration schemes because it is straightforward to compute higher order spatial derivatives by using expression (8).

\section{ARTIFICIAL DISSIPATION TERMS}

These terms are meant to stabilize the numerical convergence process when the mesh is not Cartesian, which is, unfortunately, the most common situation in industrial applications. They should be kept as simple as possible so that minimum disturbances are introduced in the computed flow field. We have implemented a second-order and a fourth-order term in the continuity equation and a fourth-order term on each of the momentum and internal energy equations. The second-order operator $L_{2 k}(\Phi)$ at node $k$, has the form:

$$
L_{2 k}(\boldsymbol{\Phi})=\sum_{i=1}^{4}\left(\Phi_{i}-\boldsymbol{\Phi}_{k}\right), \Phi \equiv \rho, u, \nu, T
$$

It is to be noted that summation in operator $L_{2 \mathrm{k}}(\Phi)$ extends only to the first four points in the cloud, not to the full six points used for the least squares approximation. We have observed that this is enough to ensure algorithm stability and, in this way, we reduce the number of operations to be performed per iteration so computational time is reduced. The second-order artificial dissipation term $O P 2(\rho)_{k}$ that we introduce in the continuity equation is

$$
O P 2(\rho)_{k}=\frac{\delta_{2 \rho}}{\Delta t} L_{2 k}(\rho)
$$

where $\delta_{2 \rho}$ is an artificial dissipation parameter and $\Delta t$ is the computational time step. The fourth-order operator $L_{4 k}(\Phi)$ at node $k$ is obtained by repeated application of operator (11). Then, fourth-order artificial dissipation terms introduced in Eqs. (1)(4), (one in each equation) are

$$
O P 4(\Phi)_{k}=\frac{\delta_{4 \Phi}}{\Delta t} L_{4 k}(\Phi), \Phi \equiv \rho, u, \nu, T
$$

where $\delta_{4 \Phi}, \Phi \equiv \rho, u, v, T$, are the artificial viscosity parameters. It is to be noted that the same artificial viscosity parameter $\delta_{4 u}$ is used for both the $x$ and $y$ momentum equations, although operators are different. Introduction of operators (11) and (12) into Eqs. (1)-(4) changes the order of these equations so that additional boundary conditions are needed.

Inflow and outflow sections:

$$
\frac{\partial}{\partial n}[O P 2(\rho)]=0, \frac{\partial}{\partial n}[O P 2(u)]=0, \frac{\partial}{\partial n}[O P 2(\nu)]=0, \frac{\partial}{\partial n}[O P 2(T)]=0
$$

Cylinder wall:

$$
\frac{\partial}{\partial n}[O P 2(\rho)]=0,[O P 2(u)]=0,[O P 2(\nu)]=0, \frac{\partial}{\partial n}[O P 2(T)]=0
$$


Operator (11) has a pseudo-Laplacian form so that, in the case of a Cartesian mesh, second- and fourth-order dissipation terms could be approximated as follows:

$$
O P 2(\Phi) \approx \frac{\delta_{2 \Phi} d^{2}}{\Delta t}\left(\frac{\partial^{2} \Phi}{\partial x^{2}}+\frac{\partial^{2} \Phi}{\partial y^{2}}\right), \quad O P 4(\Phi) \approx \frac{\delta_{4 \Phi} d^{4}}{\Delta t}\left(\frac{\partial^{4} \Phi}{\partial x^{4}}+\frac{\partial^{4} \Phi}{\partial y^{4}}\right)
$$

where $d$ is a typical distance within the cloud. Far away from the cylinder, where points are located far apart form each other, $d$ could be large. To avoid this unwanted effect that could give rise to unreasonably large values of operators (15), artificial viscosity parameters $\delta_{2 \rho}, \delta_{4 \rho}, \delta_{4 u}$, and $\delta_{4 T}$ have been made to decrease monotonically away from the cylinder. In particular, they decrease inversely proportional to the typical cloud diameter. In this way, we do not only control behavior of the equations in the far field but also decrease the total amount of artificial damping implemented in the whole computational domain.

\section{VALIDATION AND SENSITIVITY ANALYSIS}

The results published by Bijl et al. [17] have been used for validation purposes. This choice is based on the following rationale: (a) these authors used a simple geometry; i.e., a circular cylinder, (b) compressibility effects were relevant; i.e., Mach number was 0.3 , (c) they used five different meshes (number of elements ranged from 3,577 to 55,777 ) so their results could be considered grid converged, and (d) their numerical scheme was very different from the one we use since they implemented a cell centered finite volume scheme with fully implicit time integration. We have used three different computational domains, all of them of circular shape, whose main characteristics are given in Table 1 (recall that distances are made dimensionless by using the cylinder diameter). A first set of results obtained by changing grid and artificial viscosity parameters is presented in Table 2 . Aerodynamics parameters (defined in the Nomenclature section) are the same as those used in Linares and Velazquez [12]. To allow for a better comparison between different cases, the values of the following parameters, see relations (15), are included in the table:

$$
\xi_{2 p}=\frac{\delta_{2 \rho} d^{2}}{\Delta t}, \xi_{4 p}=\frac{\delta_{4 \rho} d^{4}}{\Delta t}, \xi_{4 u}=\frac{\delta_{4 i t} d^{4}}{\Delta t}, \xi_{4 T}=\frac{\delta_{4 T} d^{4}}{\Delta t}
$$

where $d$ is the minimum distance between points (see fourth line in Table 1).

The finest grid used by Bijl et al. [17] had 289 elements in the cylinder surface and the computational outer boundary was located at a distance of 20 diameters from the cylinder. Dimensionless distance between the cylinder wall and the first grid point was $1.0 \mathrm{e}-3$. For this case, Bijl et al. [17] reported $C_{-\max }=1.51$ and $S=0.244$. Our finest grid had 720 points in the cylinder surface, distance to the first node away from the wall was $4.3 \mathrm{e}-3$, and the boundary of the computational domain was located 29 diameters away from the cylinder. In these conditions, our converged results (with regard to grid and artificial viscosity parameters) were $C l_{\text {nax }}=1.56$ and $S=0.232$, which differs by a factor of 4 and $5 \%$, respectively, from the ones 
Table 1 Definition of the three grids used for validation purposes

\begin{tabular}{lccc}
\hline & Grid 1 & Grid 2 & Grid 3 \\
\hline Points in the cylinder sutface & 180 & 360 & 720 \\
Diameter of the computational domain & 58.8 & 58.8 & 58.8 \\
Total number of points & 15.780 & 18.900 & 34,860 \\
Minimum distance between points & 0.0174 & 0.0087 & 0.0044 \\
Maximum distance between points & 2.09 & 2.09 & 2.09 \\
\hline
\end{tabular}

reported by Bijl et al. [17]. Sensitivity of the results with regard to time step is presented in Table 3 . The criterion to make a fair comparison between cases has been to use a constant value of $\xi_{2 \rho}, \xi_{4 \rho^{*}} \xi_{4 \mathrm{u}}$, and $\xi_{4 \mathrm{~T}}$; see definition (16). Baseline case used for comparison has been case 11 . It can be observed in Table 3 that $C d_{\text {av }}$, $C l_{\text {max }}$, and $S$ remain basically unchanged (variations are smaller than $1 \%$ ) when the time step changes by a factor of 8 . Summarizing information provided in Tables 1,2 , and 3 , it could be said that our solution is converged with regard to the artificial viscosity parameters and with regard to spatial and temporal discretization. Grid 2 will be taken, for the remainder of this article, as the baseline.

Another test of the solution robustness is presented in Table 4. Finite Point solvers are of interest, among other aspects, because they allow for the patching-up of regions with different densities of points. As it could be expected, sharp transitions are the most sensitive zones from the standpoint of algorithm stability, and it is within these transition regions where accuracy in the prediction of derivatives is poorest and artificial damping is most needed. For instance, the baseline grid 2 was made up of three patches:

- Patch A: 21 rings of 360 points each (innermost patch: closest to the cylinder)

- Patch B: 25 rings of 180 points each (intermediate region)

- Patch C: 57 rings of 120 points each (outermost patch: farthest away from the cylinder)

In the sharp transition region from patch $\mathrm{A}$ to patch $\mathrm{B}$, the ratio of maximum to minimum distance was 2.45. A close-up view of this region is shown in the left-hand side subplot of Figure 2. Now, another computational domain was generated (grid 2-b) containing one additional very coarse narrow patch (120 points per ring) located in between patches $A$ and $B$. In this way, maximum versus minimum distance ratio within clouds in the transition region increased up to 3.70 ( $50 \%$ more than in the baseline case). A detailed view of this modified grid 2-b is shown in the right-hand side subplot of figure 2. The size of the computational domain and the total number of points were the same in both grids. Now, Table 4 presents the results obtained (cases 41 to 44 ), their comparison with baseline case 11 , and the outcome of the sensitivity study with regards to the integration time step. Table 4 shows that a $50 \%$ coarsening of the transition region caused $C d_{\text {avv }}$ to change from 1.50 to 1.40 ( $7 \%$ variation), $C l_{\max }$ from 1.46 to 1.36 (7\% variation), and $S$ from 0.235 to 0.229 (3\% variation). Again, it could be observed that the results, in the modified grid 2-b, are converged with regard to the integration time step. 
Table 2 Computed averaged drag (Cd_av). peak lift $\left(\mathrm{Cl}_{-}\right.$max $)$, and Strouhal number (S) at $\mathrm{Mach}=0.3$ and Reynolds $=1200$ for a series of different grids and artificial viscosity parameters

\begin{tabular}{|c|c|c|c|c|c|c|c|c|c|c|c|c|}
\hline Case key & 1 & 2 & 3 & 4 & 11 & 12 & 13 & 14 & 21 & 22 & 23 & 24 \\
\hline Grid & 1 & 1 & 1 & 1 & 2 & 2 & 2 & 2 & 3 & 3 & 3 & 3 \\
\hline$\Delta t \times \mathrm{le}^{4}$ & 2 & 2 & 2 & 2 & 1 & 1 & 1 & 1 & 1 & 1 & 1 & 1 \\
\hline$\delta_{2 \beta} \times 1 \mathrm{e} 4$ & 100 & 50 & 25 & 12 & 50 & 25 & 12.5 & 6.25 & 200 & 100 & 67 & 50 \\
\hline$\delta_{4 I} \times 1 \mathrm{e} 4$ & 40 & 20 & 10 & 5 & 20 & 10 & 5 & 2.5 & 80 & 40 & 30 & 20 \\
\hline$\delta_{4 T} \times \mathrm{le} 4$ & 40 & 20 & 10 & 5 & 20 & 10 & 5 & 2.5 & 80 & 40 & 30 & 20 \\
\hline$\xi_{2, \beta} \times 1 \mathrm{e} 4$ & 150 & 76 & 38 & 19 & 38 & 19 & 10 & 5 & 38 & 19 & 13 & 10 \\
\hline$\xi_{4 \rho} \times 1 \mathrm{e} 9$ & 1800 & 900 & 450 & 230 & 120 & 58 & 29 & 14 & 29 & 14 & 10 & 7.2 \\
\hline$\xi_{4 T} \times 1 \mathrm{e}^{9}$ & 1800 & 900 & 450 & 230 & 120 & 58 & 29 & 14 & 29 & 14 & 10 & 7.2 \\
\hline$C d_{-a v}$ & 1.29 & 1.35 & 1.40 & $\mathrm{FC}$ & 1.50 & 1.51 & 1.50 & $\mathrm{FC}$ & 1.55 & 1.56 & 1.56 & $\mathrm{FC}$ \\
\hline $\mathrm{Cl}_{-\max }$ & 1.15 & 1.28 & 1.37 & $\mathrm{FC}$ & 1.46 & 1.51 & 1.50 & $\mathrm{FC}$ & 1.50 & 1.58 & 1.57 & $\mathrm{FC}$ \\
\hline$s^{-}$ & 0.227 & 0.238 & 0.240 & $\mathrm{FC}$ & 0.235 & 0.232 & 0.229 & $\mathrm{FC}$ & 0.229 & 0.234 & 0.232 & $\mathrm{FC}$ \\
\hline \multicolumn{13}{|c|}{ Bijl et al. [17], finest grid } \\
\hline$C d_{-a v}$ & & & & & & & & & & & & Not available \\
\hline $\mathrm{Cl}_{-\max }$ & & & & & & & & & & & & 1.51 \\
\hline$S^{-}$ & & & & & & & & & & & & 0.244 \\
\hline
\end{tabular}

Results reported by Bijl et al. [17] are included for comparison purposes. 
Table 3 Sensitivity of the results with regard to the computational time step

\begin{tabular}{lrrrrr}
\hline Case key & 11 & 31 & 32 & 33 & 34 \\
Grid & 2 & 2 & 2 & 2 & 2 \\
$\Delta t \times 1 \mathrm{e} 4$ & 1 & 2 & 4 & 8 & 16 \\
$\delta_{2 \rho} \times 1 \mathrm{e} 4$ & 50 & 100 & 200 & 400 & 800 \\
$\delta_{4 \rho} \times 1 \mathrm{e} 4$ & 20 & 40 & 80 & 160 & 320 \\
$\delta_{4 u} \times 1 \mathrm{e} 4$ & 20 & 40 & 80 & 160 & 320 \\
$\delta_{4 T} \times 1 \mathrm{e} 4$ & 20 & 40 & 80 & 160 & 320 \\
$\xi_{2 \rho} \times 1 \mathrm{e} 4$ & 38 & 120 & 120 & 38 & 38 \\
$\xi_{4 \rho} \times 1 \mathrm{e} 9$ & 120 & 120 & 120 & 120 & 120 \\
$\xi_{4 u} \times 1 \mathrm{e} 9$ & 120 & 120 & 120 & 120 & 120 \\
$\xi_{4 T} \times$ le9 & 120 & 1.50 & 1.50 & 1.50 & 120 \\
$C d_{\text {av }}$ & 1.50 & 1.46 & 1.47 & 1.47 & $\mathrm{FC}$ \\
$\mathrm{Cl}$ & 1.46 & 0.233 & 0.233 & 0.234 & $\mathrm{FC}$ \\
$S$ & 0.235 & & & $\mathrm{FC}$ \\
\hline
\end{tabular}

Table 4 Sensitivity of the results with regard to grid non-homogeneity and time step

\begin{tabular}{lrrrrr}
\hline Case key & 11 & 41 & 42 & 43 & 44 \\
Grid & 2 & $2-\mathrm{b}$ & $2-\mathrm{b}$ & $2-\mathrm{b}$ & $2-\mathrm{b}$ \\
$\Delta t \times 1 \mathrm{e} 4$ & 1 & 1 & 2 & 4 & 8 \\
$\delta_{2 \rho} \times 1 \mathrm{e} 4$ & 50 & 50 & 100 & 200 & 400 \\
$\delta_{4 \rho} \times 1 \mathrm{e} 4$ & 20 & 20 & 40 & 80 & 160 \\
$\delta_{4 t} \times 1 \mathrm{e} 4$ & 20 & 20 & 40 & 80 & 160 \\
$\delta_{4 T} \times 1 \mathrm{e} 4$ & 20 & 20 & 40 & 80 & 160 \\
$\xi_{2 \rho} \times 1 \mathrm{e} 4$ & 38 & 38 & 38 & 38 & 38 \\
$\xi_{4 \rho} \times 1 \mathrm{e} 9$ & 120 & 120 & 120 & 120 & 120 \\
$\xi_{4 u} \times 1 \mathrm{e} 9$ & 120 & 120 & 120 & 120 & 120 \\
$\xi_{4 T} \times 1 \mathrm{e} 9$ & 120 & 1.40 & 120 & 120 & 120 \\
$C d_{\text {av }}$ & 1.50 & 1.36 & 1.40 & 1.40 & 1.40 \\
$C l_{\text {-max }}$ & 1.46 & 0.229 & 0.229 & 1.37 & 1.37 \\
$S^{-23}$ & 0.235 & & & 0.229 & 0.229 \\
\hline
\end{tabular}
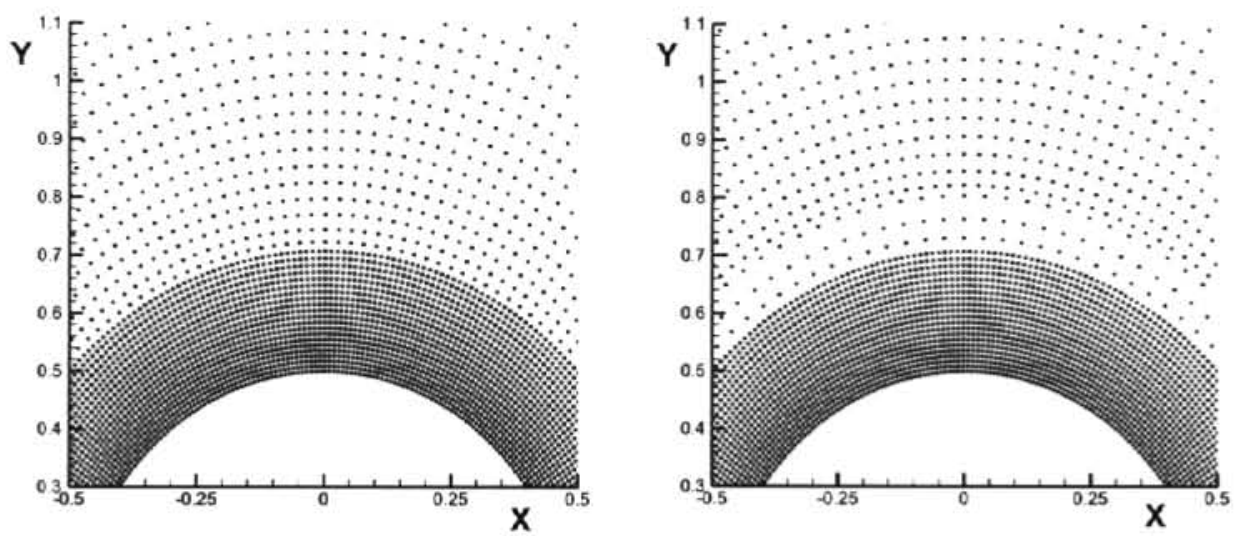

Figure 2. Close-up view of the points distribution in the baseline grid 2 (left) and in the modified grid 2-b (right). 


\section{RESULTS}

The flow parameters that have been considered are Mach 0.4 and Reynolds 150 . An ideal fluid has been modeled so that properties such as viscosity and thermal conductivity are assumed to be constant. This is certainly not appropriate when temperature variations are large, but this application case focuses on having a preliminary understanding of the role that thermal energy and kinetic energy coupling play on vortex shedding behavior. All computation cases to be addressed in this section use grid 2 (see Table 1). Computational time step and artificial dissipation parameters are those used for previous case 11 (see Table 2).

\section{First Set of Results}

We start by implementing a constant temperature boundary condition:

$$
T_{w}(\theta, t)=a_{0}, \quad \forall \theta, t
$$

The azimuth angle $\theta$ is measured counterclockwise from the positive horizontal axis (as in standard cylindrical coordinates, see Figure 1). The results obtained for the different temperature levels are presented in Table 5. It could be observed that $C d$ depends very weakly on the wall temperature while $\mathrm{Cl}$ changes by a factor of the order of $\pm 30-40 \%$ when the wall temperature is either doubled or halved. The variation on the Strouhal number is also small. Table 6 shows the results obtained when using a time dependent wall temperature defined as follows:

$$
T_{w}(t)=b_{0}+b_{1} \sin (2 \pi t 2 S)+b_{2} \sin (2 \pi t S), \quad \forall \theta
$$

Table 5 Results obtained for constant wall temperatures

\begin{tabular}{llllll}
\hline Case key & 51 & 52 & 53 & 54 & 55 \\
\hline$a_{0}$ & 0.50 & 0.75 & 1.00 & 1.25 & 1.50 \\
$C d_{\text {ave }}$ & 1.45 & 1.45 & 1.44 & 1.42 & 1.39 \\
$C d_{\text {span }}$ & 0.04 & 0.03 & 0.03 & 0.02 & 0.02 \\
$C d_{\text {-max }}$ & 0.73 & 0.64 & 0.56 & 0.48 & 0.40 \\
$S$ & 0.179 & 0.177 & 0.175 & 0.171 & 0.166 \\
\hline
\end{tabular}

Table 6 Results obtained when the prescribed thermal oscillation depends only on time

\begin{tabular}{lcccccccccccc}
\hline $\begin{array}{l}\text { Case } \\
\text { key }\end{array}$ & 61 & 62 & 63 & 64 & 65 & 66 & 71 & 72 & 73 & 74 & 75 & 76 \\
\hline$b_{0}$ & 0.75 & 0.75 & 1.00 & 1.00 & 1.25 & 1.25 & 0.75 & 0.75 & 1.00 & 1.00 & 1.25 & 1.25 \\
$b_{1}$ & 0 & 0 & 0 & 0 & 0 & 0 & -0.25 & -0.50 & -0.25 & -0.50 & -0.25 & -0.50 \\
$b_{2}$ & -0.25 & -0.50 & -0.25 & -0.50 & -0.25 & -0.50 & 0 & 0 & 0 & 0 & 0 & 0 \\
$C d_{-}$ave & 1.43 & 1.43 & 1.43 & 1.43 & 1.42 & 1.42 & 1.47 & 1.47 & 1.46 & 1.45 & 1.43 & 1.43 \\
$C d_{-}$span & 0.08 & 0.13 & 0.05 & 0.08 & 0.05 & 0.08 & 0.11 & 0.24 & 0.08 & 0.14 & 0.07 & 0.13 \\
$C d_{-\max }$ & 0.60 & 0.59 & 0.55 & 0.54 & 0.48 & 0.47 & 0.64 & 0.64 & 0.58 & 0.58 & 0.50 & 0.50 \\
$S$ & 0.177 & 0.177 & 0.175 & 0.175 & 0.171 & 0.171 & 0.177 & 0.177 & 0.175 & 0.175 & 0.171 & 0.171
\end{tabular}


$S$ is the vortex shedding Strouhal number of the corresponding constant temperature case (Strouhal number of Table 5). The forcing term associated to $b_{1}$ in $\mathrm{Eq}$. (18) has the drag frequency $2 S$, while the term associated to $b_{2}$ has the lift frequency $S$. The results obtained could be summarized as follows:

- The actual level of the constant wall temperature boundary condition (cases 51 to 55) has a weak influence on drag and a strong influence on lift. Temperature changes of the order of $\pm 50 \%$ cause $C d$ and $C l$ variations of the order $\pm 2 \%$ and $\pm 30 \%$, respectively. Strouhal number variations are also small (of the order of $\pm 3 \%$ ).

- A wall temperature that pulsates at the lift frequency (cases 61 to 66) has rather small influence on both the average drag and the maximum lift, even if the amplitude of the pulsation is large (of the order of $50 \%$ of the mean value). We observe, in these cases, that changes on $\mathrm{Cd}$ are smaller than $2 \%$, while changes on $\mathrm{Cl}$ are of the order of $6 \%$ at most.

- When the wall temperature pulsates at the drag frequency (cases 71 to 76 ) the influence on average drag and maximum lift is also small. However, in this case, $C d$ variations around its average value grow larger and are in the range of $\pm 16 \%$ (case 72 ) to $\pm 9 \%$ (case 76 ). It is to be noted that the amplitude of the $C d$ oscillations in the cases with constant wall temperature (cases 51 to 55 ) was $2 \%$ at most.

These results suggest that lift control requires, in addition to time, a spatial $(\theta)$ dependent temperature variation. In particular, the idea is to pulsate in opposite phase at the top and bottom of the cylinder to enhance the alternating vertical force generated by vortex shedding.

\section{Second Set of Results}

In accordance to the previous conclusion, we have now chosen the following space- and time-dependent functional behavior for the cylinder wall temperature (cases 81 to 88 in Table 7):

$$
T_{w}(\theta, t)=1+c_{1} \sin \left(2 \pi t 2 S_{b}\right) \cos (\theta)+c_{2} \sin \left(2 \pi t S_{b}\right) \sin (\theta)
$$

where we restrict ourselves to the case of average temperature equal to 1 and select $S_{b}$ $=0.175$, which is the vortex shedding Strouhal number of baseline case 53 with no thermal oscillation present (see Table 5).

The following aspects regarding implementation of boundary condition (19) should be noted: (a) Computation of all cases 81 to 88 has started from the solution of

Table 7 Results obtained when the prescribed thermal oscillation depends on both time and azinuth angle

\begin{tabular}{lccccccccc}
\hline Case key & 53 & 81 & 82 & 83 & 84 & 85 & 86 & 87 & 88 \\
\hline$c_{1}$ & 0 & 0.5 & -0.5 & 0 & 0 & 0.5 & 0.5 & -0.5 & -0.5 \\
$c_{2}$ & 0 & 0 & 0 & 0.5 & -0.5 & 0.5 & -0.5 & 0.5 & -0.5 \\
$T_{\min }$ & 1 & 0.5 & 0.5 & 0.5 & 0.5 & 0.375 & 0.375 & 0.375 & 0.375 \\
$T_{\max }$ & 1 & 1.5 & 1.5 & 1.5 & 1.5 & 1.625 & 1.625 & 1.625 & 1.625 \\
$C d_{\text {-ave }}$ & 1.44 & 1.45 & 1.45 & 1.45 & 1.45 & 1.44 & 1.44 & 1.47 & 1.44 \\
$C d_{\text {-span }}$ & \pm 0.03 & \pm 0.22 & \pm 0.22 & \pm 0.02 & \pm 0.02 & \pm 0.22 & \pm 0.22 & \pm 0.19 & \pm 0.17 \\
$C$ _-max $_{\text {_ma }}$ & 0.56 & 0.62 & 0.62 & 0.74 & 0.74 & 0.77 & 0.77 & 0.77 & 0.77 \\
$\mathrm{~S}$ & 0.175 & 0.175 & 0.175 & 0.175 & 0.175 & 0.175 & 0.175 & 0.175 & 0.175 \\
\hline
\end{tabular}




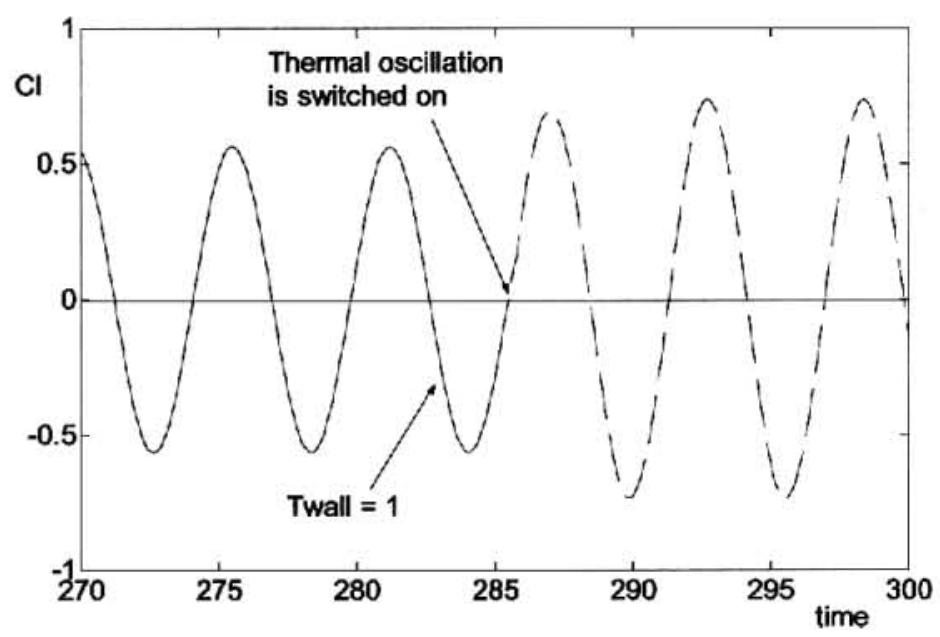

Figure 3. Illustration of the way chosen to switch the thermal oscillation on.

baseline case 53 . The thermal oscillation on the cylinder surface was switched on when the lift coefficient $\mathrm{Cl}$ in the upwards stroke was zero (see Figure 3 ). In this way we ensure that $T_{\text {wall }}=1$ when the thermal oscillation starts. (b) The term that contains $\cos (\theta)$ reaches its maximum and minimum values at $\theta=0, \pi$, oscillates at the drag force baseline frequency $2 S_{b}$, and modifies temperature mostly in the forward and backward regions of the micro-cylinder where pressure contribution to drag is higher. (c) The term that contains $\sin (\theta)$ reaches its maximum and minimum values at $\theta=\pi / 2,3 \pi / 2$, oscillates at the lift force baseline frequency $S_{b}$, and modifies temperature mostly in the upper and lower regions of the micro-cylinder where pressure contribution to lift is higher.

The results obtained are presented in Table 7. $T_{\min }$ and $T_{\max }$ are the minimum and maximum local wall temperatures obtained for the different combinations of the $c_{1}$ and $c_{2}$ parameters. Regarding these results, the following observations could be made:

- Cases 81 and 82: surface temperature oscillation depends on $\cos (\theta)$ only. The amplitude of the $C d$ oscillation is \pm 0.22 now, while this amplitude was \pm 0.03 in the baseline constant temperature case 53 and \pm 0.14 in case 74 with only time-dependent pulsation present. The time-averaged drag $C d_{\text {ave }}$ did not change noticeably. The maximum lift $\mathrm{Cl}_{\max }$ increased to 0.62 now from the baseline value of $0.56(11 \%$ up). The vortex shedding Strouhal number remained unaffected by the surface thermal oscillation.

- Cases 83 and 84: surface temperature oscillation depends on $\sin (\theta)$ only. The amplitude of the drag coefficient $\mathrm{Cd}$ was not affected by this thermal oscillation. The time averaged drag $\mathrm{Cd}_{-}$ave was not affected either. The maximum lift $\mathrm{Cl}_{-}$max was 0.74 , up from 0.56 in the baseline case ( $32 \%$ increase). The Strouhal number was not affected by the thermal oscillation.

- Cases 85 to 88: surface temperature oscillation depends on both $\cos (\theta)$ and $\sin (\theta)$. The amplitude of the drag coefficient $C d$ varied between \pm 0.17 and \pm 0.22 . The time averaged drag $C d_{\text {ave }}$ was not affected by this thermal oscillation. The maximum lift $\mathrm{Cl}_{\max }$ was 0.77 , which represents a $38 \%$ increase over the baseline case. The Strouhal number was not affected by the thermal oscillation. 
When the prescribed surface temperature oscillates with the frequency of the baseline lift and drag, aerodynamics forces can be modulated. In particular, lift amplitude could be increased by a factor of $38 \%$ (from 0.56 to 0.77 ) and drag could be made to oscillate with the amplitude of \pm 0.22 around its mean value of 1.44 (that is a variation of $\pm 15 \%$ ). In all these cases, the observed vortex shedding frequency did not change when compared to the vortex shedding baseline frequency.

We now investigate how sensitive the behavior just described is with regard to the local spatial details of the prescribed pulsation. To do so, we compute an additional case with the following boundary condition:

$$
\begin{array}{ll}
\frac{\partial T_{w}}{\partial n}=0, & -\frac{\pi}{4} \leq \theta \leq \frac{\pi}{4} \\
T_{w}(\theta, t)=1-0.5 \sin \left(2 \pi t S_{b}\right), & \frac{\pi}{4}<\theta<\frac{3 \pi}{4} \\
\frac{\partial T_{w}}{\partial n}=0, & -\frac{3 \pi}{4} \leq \theta \leq \frac{5 \pi}{4} \\
T_{w}(\theta, t)=1+0.5 \sin \left(2 \pi t S_{b}\right), & \frac{5 \pi}{4}<\theta<\frac{7 \pi}{4}
\end{array}
$$

This boundary condition is a crude representation of the one used for case 84 . In particular, we now pulsate with opposite time phase and constant spatial distribution at the top and bottom of the micro-cylinder while keeping adiabatic the forward and backward stagnation regions. In this case we obtain $C d=1.45 \pm 0.02, C l_{-\max }=0.72$, and $S=0.175$, while the corresponding results of case 84 were $C d=\overline{1} .45 \pm 0.02$, $C l_{\max }=0.74$, and $S=0.175$. Then, it could be said that the behavior of the aerodynamics coefficients (that are of a global nature) depends very weakly on the local details of the prescribed pulsation.

\section{Third Set of Results}

We now study the time evolution of surface pressure after the thermal oscillation has been switched on. As it has been said before, all computations 81 to 88 start from baseline computation 53 at the time instant when $C l$ is zero and stroking upwards (see Figure 3). Let us consider cases 83 and 84 , which behave in a different way in the time instants that follow the switch from constant to pulsating wall temperature. In particular, temperature and pressure in case 83 tend to increase at the top of the cylinder $(\theta=\pi / 2)$ while decreasing at the bottom $(\theta=3 \pi / 2)$. That is, thermal oscillation 83 tends, in the initial time instants, to decrease $C l$ which is precisely the opposite of what the fluid is doing at that very moment. On the contrary, thermal oscillation in case 84 tends to increase $\mathrm{Cl}$ in phase with fluid behavior. Figure 4 shows the time evolution of $\mathrm{Cl}$ for cases 83 (solid line) and 84 (dashed line) after the temperature oscillation has been switched on. In this picture it could be observed that case 83 performs 17 vortex shedding cycles within the time span of 100 characteristic times while case 84 performs 18 cycles. The time history of the Strouhal number for case 83 (downwards pointing triangles) and case 84 (upwards pointing triangles) is presented in Figure 5. There it can be seen that after about 15 cycles, vortex shedding in both cases 83 and 84 occurs at the same frequency (Strouhal number equal to the baseline value of 0.175 ). However, case 83 adjusts itself by passing through a series of shedding cycles characterized by a smaller Strouhal number. 


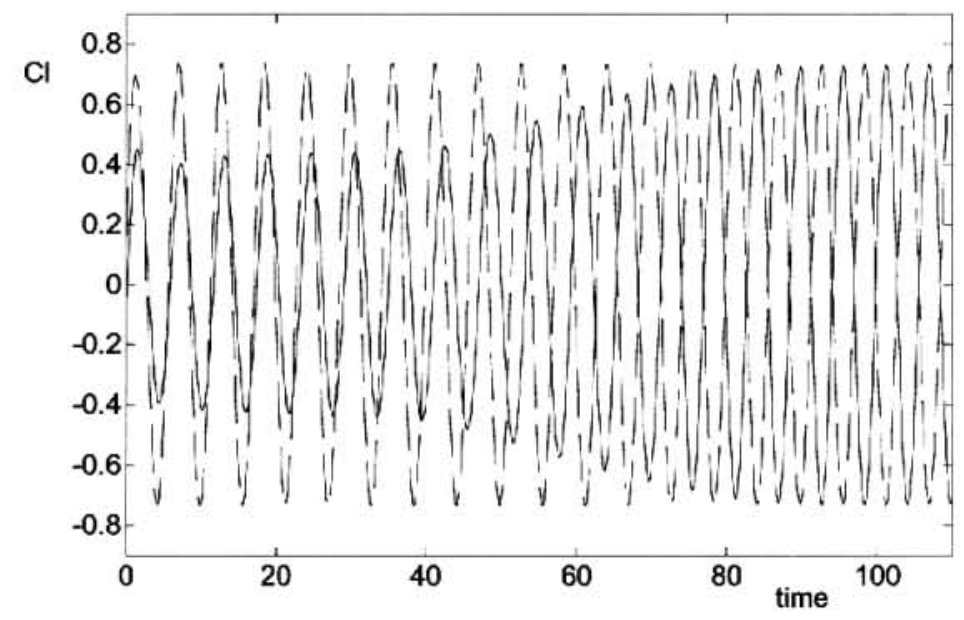

Figure 4. Time evolution of $\mathrm{Cl}$ for case 83 (solid line) and 84 (dashed line).

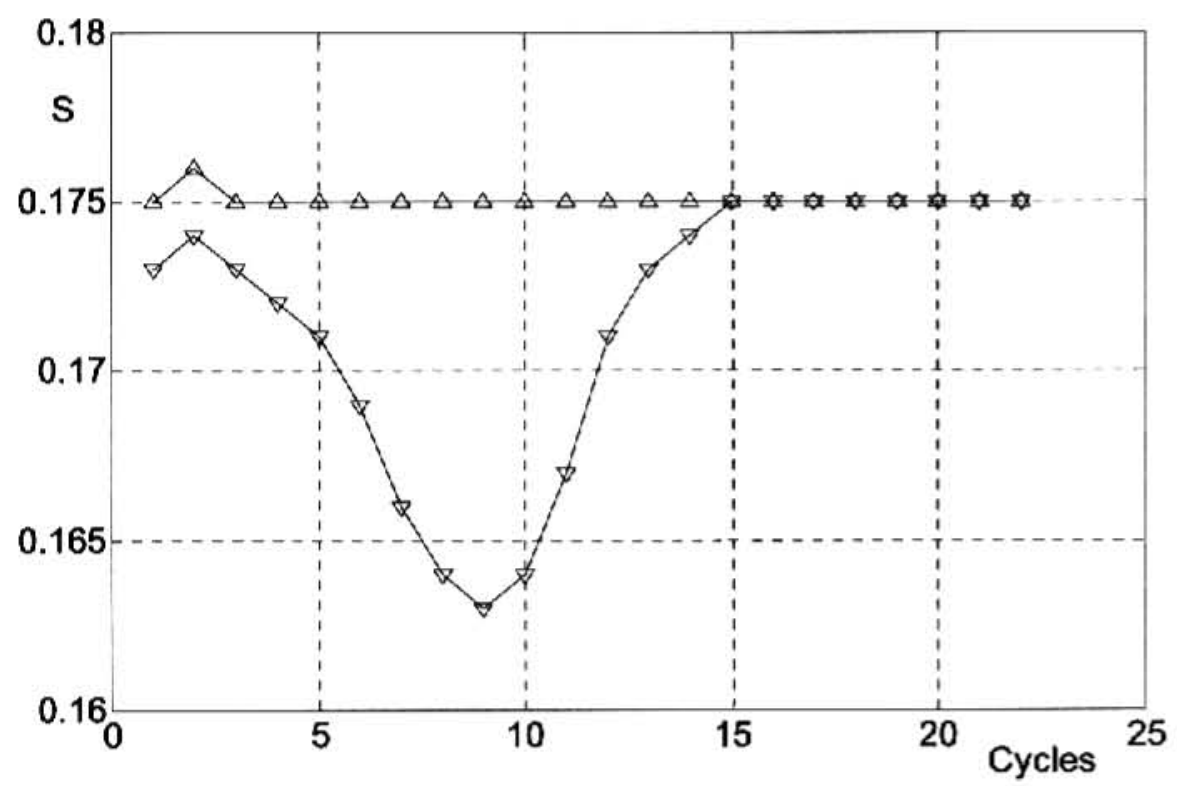

Figure 5. Time history of the Strouhal number for case $83(\nabla)$ and $84(\Delta)$.

Figures 6 (case 83) and 7 (case 84) show the time evolution of wall temperature, density, pressure, and $\mathrm{Cl}$. Values at the top of the cylinder $(\theta=\pi / 2)$ are shown in the left-hand side subplots, while results at the bottom $(\theta=3 \pi / 2)$ are presented in the right-hand side subplots. $\mathrm{Cl}$ reaches its maximum value in the oscillatory steady-state situation when pressure at the top of the cylinder $(\theta=\pi / 2)$ is minimum. This minimum value of pressure is achieved by a combination of minimum temperature and maximum density (see left-hand side subplots of Figures 6 and 7). The opposite behavior 

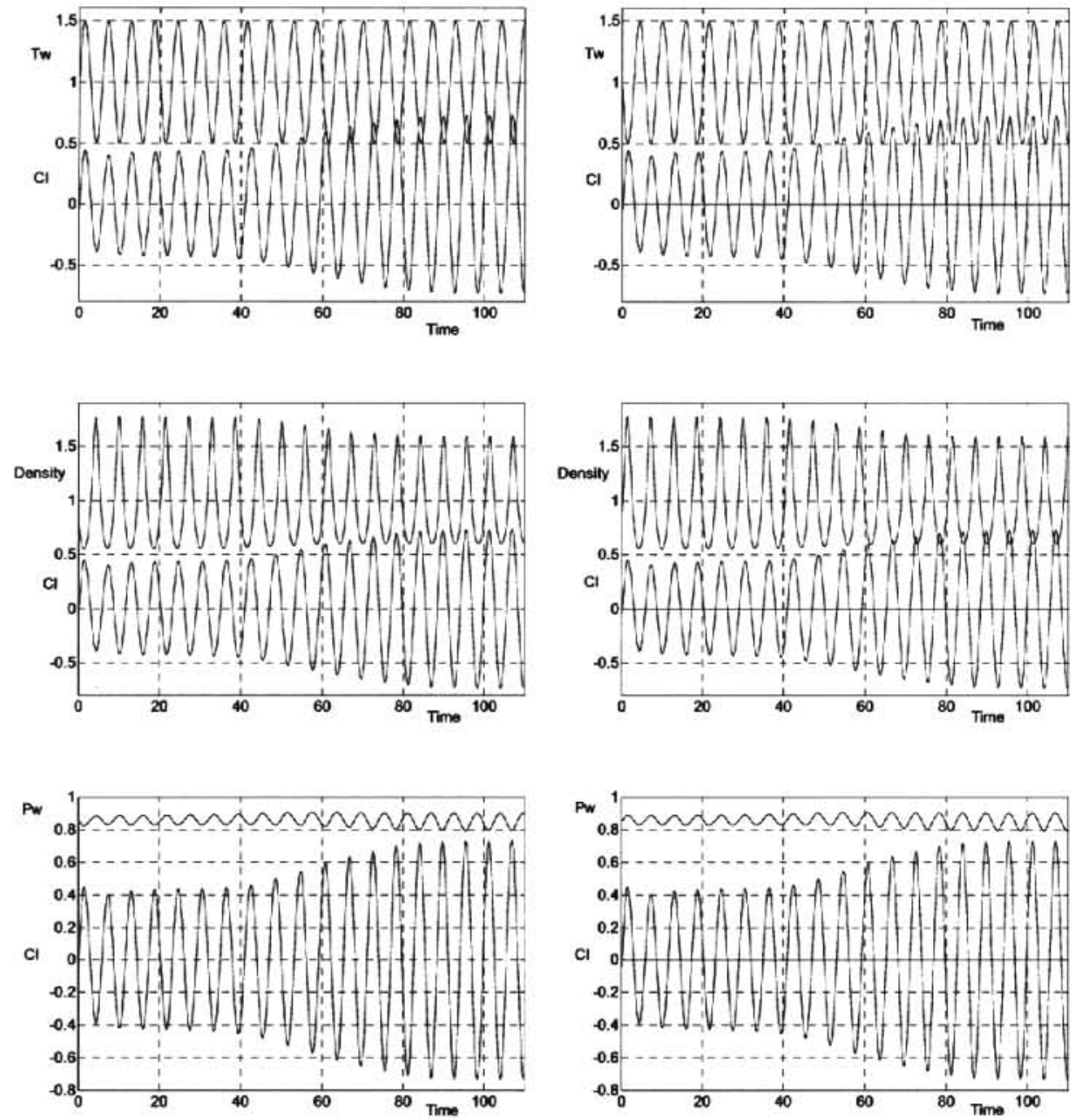

Figure 6. Case 83. Time evolution of $\mathrm{Cl}, T_{\text {wall }}, \rho_{\text {wall }}$, and $P_{\text {wall }}$. Left-hand side plots: $\theta=\pi / 2$. Right-hand side plots: $\theta=3 \pi / 2$.

happens at the bottom of the cylinder $(\theta=3 \pi / 2)$. Regarding the initial time instants of the oscillatory transient state, the temperature boundary condition of case 83 tends to generate a combination of maximum temperature and minimum density at the top of the cylinder that is the opposite of the long-term behavior. This means that the fluid needs to adjust itself during the oscillatory transient state. The adjustment comes in the shape of a (already mentioned) continuously changing vortex shedding frequency.

\section{Fourth Set of Results}

In cases 81 to 88 , the wall temperature oscillation frequency was prescribed to be equal to the frequency of the baseline lift and drag (case 53). Now, we study flow 

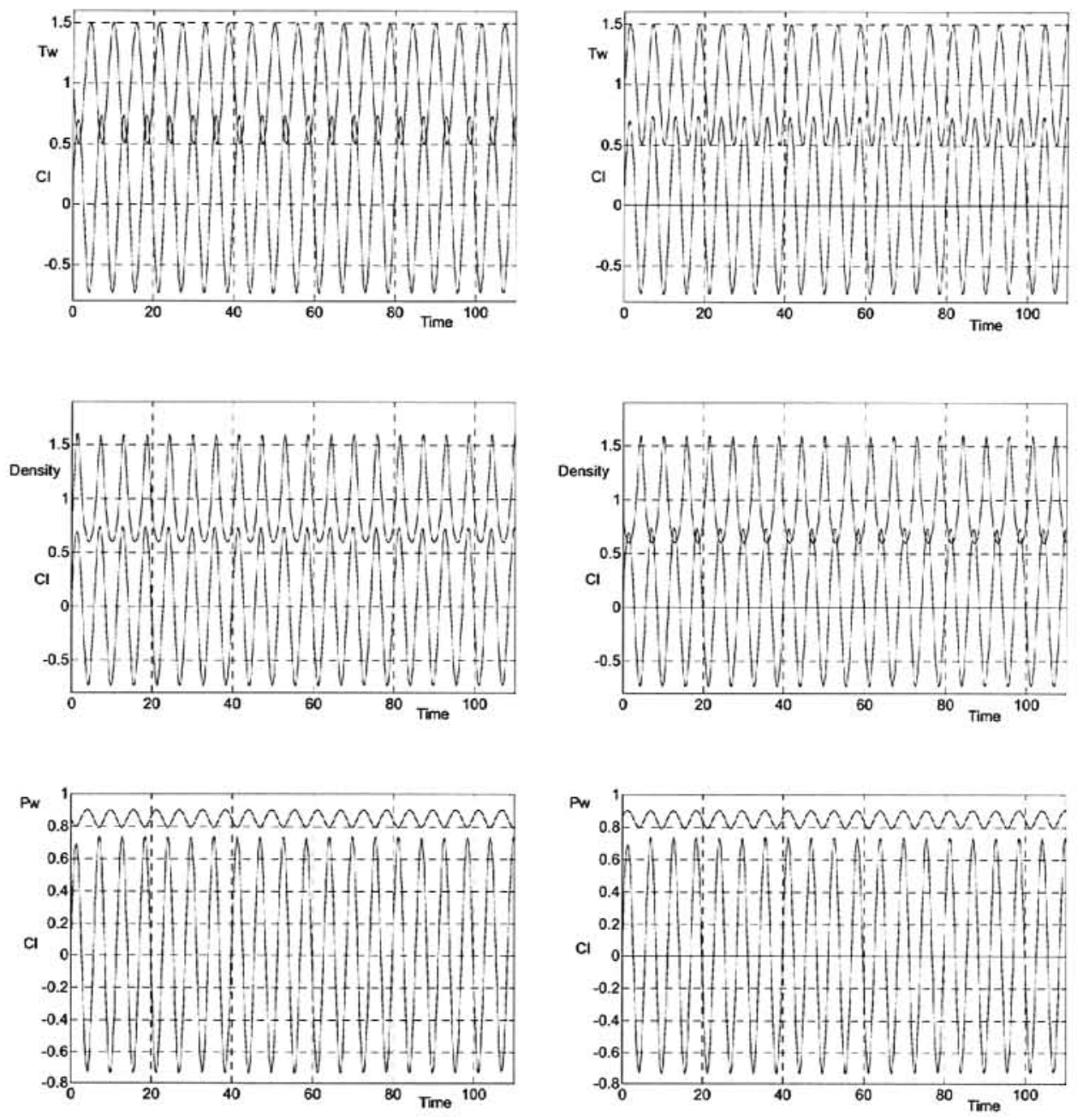

Figure 7. Case 84. Time evolution of $\mathrm{Cl}, T_{\text {wall }}, \rho_{\text {wall }}$, and $P_{\text {wall }}$. Left-hand side plots: $\theta=\pi / 2$. Right-hand side plots: $\theta=3 \pi / 2$.

behavior when this is no longer the case. In particular, we prescribe the thermal oscillation as follows:

$$
T_{w}(\theta, t)=1-05 \sin \left(2 \pi t \mathrm{~S}_{\mathrm{b}} \mathrm{F}\right) \sin (\theta)
$$

where $S_{b}=0.175$ as before and $F$ is a parameter that assumes the following values: $1.20,1.10,0.91,0.83,0.71$, and 0.56 (see Table 8 ). The Strouhal number history for all these cases is presented in the six subplots of Figure 8 . We computed 500 characteristics times in each simulation and this amounts to a number of vortex shedding cycles ranging from 80 to 90 depending on the case. The baseline Strouhal number $S_{b}=0.175$ is plotted as a dash-dotted horizontal line in each subplot of Figure 8. The average Strouhal number for the last 60 cycles of each case is plotted as a solid horizontal line. Regarding these results, the following observations could be made: 
Table 8 Results obtained when the frequency of the thermal oscillation is allowed to change

\begin{tabular}{|c|c|c|c|c|c|c|}
\hline Case key & 91 & 92 & 93 & 94 & 95 & 96 \\
\hline$F$ & 1.2 & 1.1 & $1 / 1.1$ & $1 / 1.2$ & $1 / 1.4$ & $1 / 1.8$ \\
\hline$S_{b} \times F$ & 0.210 & 0.193 & 0.159 & 0.145 & 0.124 & 0.098 \\
\hline$S_{\mathrm{av}}$ & 0.177 & 0.180 & 0.159 & 0.172 & 0.174 & 0.174 \\
\hline$S_{\text {span }}$ & $0.171-0.184$ & $0.167-0.188$ & 0 & $0.160-0.185$ & $0.163-0.189$ & $0.160-0.190$ \\
\hline
\end{tabular}
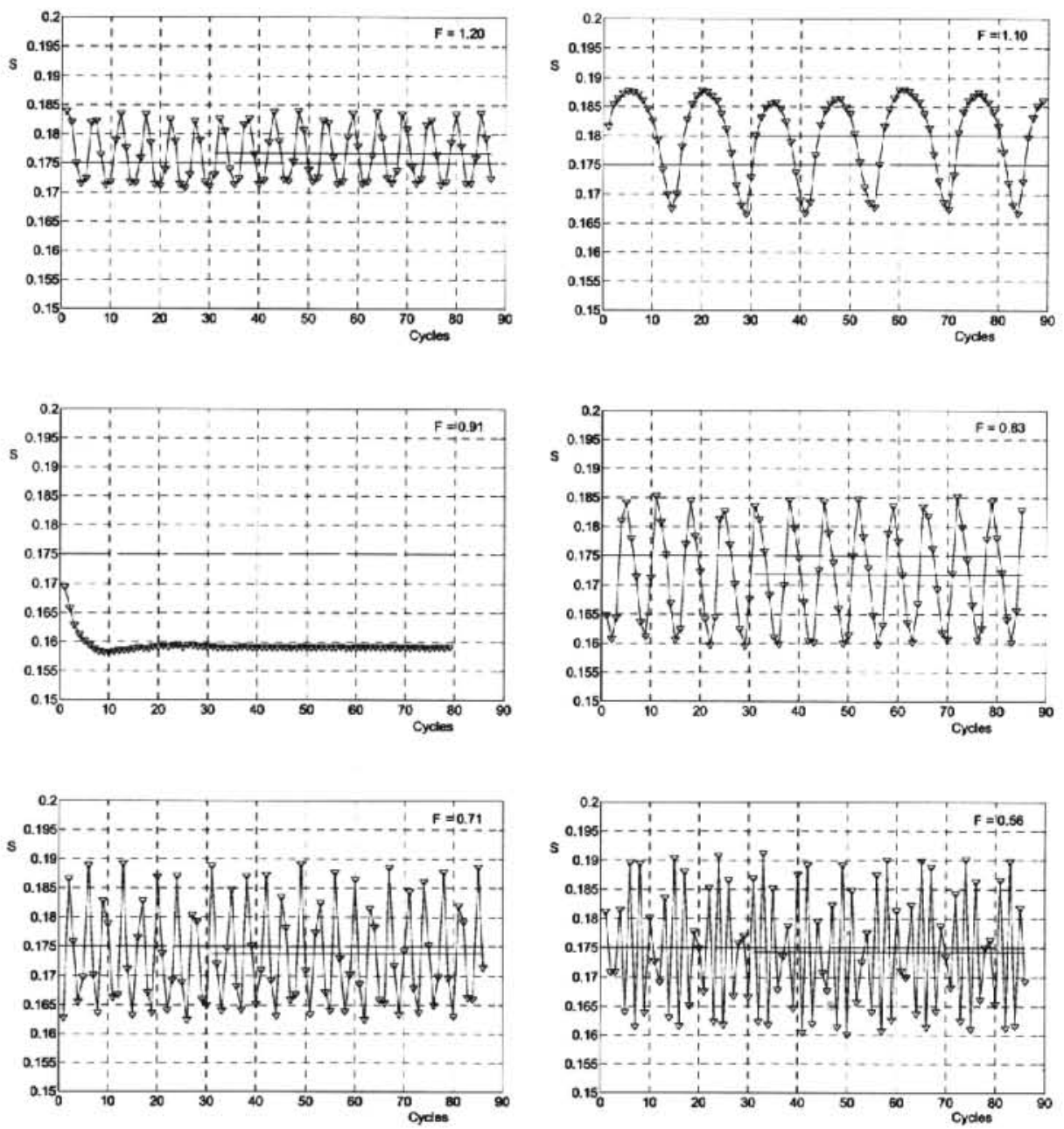

Figure 8. Time evolution of the Strouhal number for different values of the prescribed thermal oscillation frequency.

- Cases with $F<1$. If $F$ is sufficiently close to 1 (for instance, case 93 with $F=0.91$ ), the vortex shedding frequency follows the frequency of the prescribed thermal oscillation. In this case there is a full coupling between the vortex shedding 
frequency and the frequency of the forcing thermal oscillation. Smaller values of $F$ lead to a different dynamic behavior; i.e., the vortex shedding frequency is not constant and it changes from one cycle to the next. For case $94(F=0.83)$, time evolution of the Strouhal number follows a regular pattern around its mean value. For case 95 ( $F=0.71)$, the pattern loses regularity and, finally, for case 96 ( $F=$ $0.56)$, the pattern becomes highly irregular. The average value of the time-dependent Stroudhal number depends on $F$. When $F$ moves away from its baseline value of 1 , the average Strouhal number also moves away from the baseline value of 0.175 . However, if $F$ keeps decreasing, the average Strouhal number bounces back and gets closer to the baseline value. For cases with $F=0.91,0.83,0.71$, and 0.56 (see Table 8) the average Strouhal numbers are $0.159,0.172,0.174$, and 0.174 , respectively. That is, when the forcing and baseline frequencies are strongly decoupled, the fluid reverts, on the average, to its natural vortex shedding frequency.

- Cases with $F>1$. We have found that these cases behave qualitatively like the ones already described with $F<1$. The main difference is that Strouhal number follows a more regular pattern and the amplitude of the Strouhal oscillation is smaller.

Summarizing, forcing a temperature oscillation whose frequency is different from the natural (baseline) vortex shedding frequency leads to a variety of different flow patterns. When the decoupling is weak, vortex shedding frequency follows the frequency of the thermal forcing oscillation. When differences between both frequencies are large, the flow tends, on the average, to revert back to its natural vortex shedding frequency but the changes from cycle to cycle are large.

\section{CONCLUSIONS}

We found that the prescribed spatial and temporal thermal oscillations at the surface of a micro-cylinder have a significant effect on the flow field behavior. This behavior suggests that it might be worthwhile to explore the possibility of using thermal oscillations in MEMS to exert some kind of control on aerodynamics forces and vortex shedding frequencies.

Lift and drag can be modulated to some extent when the frequency of the prescribed thermal oscillation is the same as the natural vortex shedding frequency of the problem with constant wall temperature. In some cases, we obtained values of $\mathrm{Cl}_{\max } 38 \%$ larger than in the baseline case and were able to generate $C d$ variations of \pm 0.22 that are much larger than the corresponding baseline variations $( \pm 0.03)$.

When frequency of the forcing thermal oscillation is different from the natural vortex shedding frequency of the problem, we found that two different types of behavior appear. If both frequencies are close to each other, vortex shedding takes place at the forcing thermal frequency (both phenomena are strongly coupled). If these two frequencies are located far apart, vortex shedding frequency changes from one cycle to the next and the average Strouhal number gets closer to the natural frequency (both phenomena are weakly coupled). 


\section{REFERENCES}

1. J.A. Pelesko, and D.H. Bernstein. Modelling MEMS and NEMS, Chapman and Hall/CRC. New York. 2003.

2. R.M. Kirby, G.E. Karniadakis, O. Mikulchenko, and K. Mayaran, Integrated Simulation for MEMS: Coupling Flow-Structure-Thermal-Electrical Domains, in M. Gad-el-Hak, ed., The MEMS Handbook, CRC Press, New York, pp. 5-1, 5-25, 2002.

3. H. Iwai. T. Mambo. N. Yamamoto, and K. Suzuki. Laminar Convective Heat Transfer from a Circular Cylinder Exposed to a Low Frequency Zero-Mean Velocity Oscillating Flow. Intemational Journal of Heat and Mass Transfer, vol. 47. pp. 4659-4672, 2004.

4. F.M. Malifouz, and H.M. Badr. Forced Convection from a Rotationally Oscillating Cylinder Placed in a Uniform Stream. International Journal of Heat and Mass Transfer, vol. 43, pp. 3093-3104, 2000.

5. C. Gau. S.X. Wu, and H.S. Su. Synchronisation of Vortex Shedding and Heat Transfer Enhancement over a Heated Cylinder Oscillating with Small Amplitude in the Streamwise Direction. Joumal of Heat Transfer. vol. 123, no. 6, pp. 1139-1148, 2001.

6. A.B. Wang, Z. Travnicek, and K.C. Chia. On the Relationship of Effective Reynolds Number and Struhal Number for the Laminar Vortex Shedding of a Heated Circular Cylinder, Physics of Fluids, vol. 12, no. 6, pp. 1401-1410, 2000.

7. X.R. Zhang, S. Maruyama, and S. Sakai, Numerical Investigation of Laminar Natural Convection on a Heated Vertical Plate Subjected to a Periodic Oscillation, International Journal of Heat and Mass Transfer, vol. 47, pp. 4439-4448, 2004.

8. E. Ramos, B.D. Storey, F. Sierra, R. Zuniga, and A. Avramenko, Temperature Distribution in an Oscillatory Flow with a Sinusoidal Wall Temperature. International Joumal of Heat and Mass Transfer, vol. 47, pp. 4929-4938. 2004.

9. D.G. Kang, and J.M. Hyun, Bouyant Convection in an Enclosure under Time-Periodic Magnetizing Force, Intertational Joumal of Heat and Mass Transfer, vol. 50. pp. 605-615, 2007.

10. Z.Y. Guo, and X.B. Wu. Compressibility Effect on the Gas Flow and Heat Transfer in a Microtube. International Journal of Heat and Mass Transfer, vol. 40, pp. 32513254. 1997.

11. Z.Y. Guo, and X.B. Wu, Further Study on Compressibility Effects on the Gas Flow and Heat Transfer in a Microtube, Microscale Thermophysical Engineering, vol. 2, pp. 111-120, 1998.

12. B. Linares, and A. Velazquez. Heat Transfer Effects on Unsteady. Compressible, Laminar Flow around Microcylinders. Nanoscale and Microscale Thermophysical Engineering. vol. 10. pp. 305-320, 2006.

13. V.N. Dobrovolsky, L.V. Ishchuk, G.K. Ninidze, M. Baculani, A. Ferrari, and G. Lamedica, High-Amplitude High-Frequency Oscillations of Temperature, ElectronHole Pair Concentration, and Current in Silicon-on-Insulator Structures. Joumal of Applied Physics, vol. 88, no. 11, 6554-6559, 2000.

14. C. Hirsch, Numerical Computation of External and Internal Flows, John Wiley \& Sons, Chichester, 1994.

15. B. Mendez, and A. Velazquez, Finite Point Solver for the Simulation of 2-D Laminar, Incompressible Unsteady Flows, Computer Methods in Applied Mechanics and Engineering, vol. 159 , pp. 383-394, 2004.

16. B. Mendez, and A. Velazquez. Finite Point Based Numerical Study on the Unsteady Laminar Wake behind Square Cylinders. International Journal of Numerical Methods for Heat and Fluid Flow, vol. 17, pp. 108-132, 2007.

17. H. Bijl. M. Carpenter, V.N. Vatsa, and C.A. Kennedy, Implicit Time Integration Schemes for the Unsteady Compressible Navier-Stokes Equations: Laminar Flow, Journal of Computational Physics, vol. 179. pp. 313-329. 2002. 